\title{
Phase I/II Study of Intrathecal Administration of Recombinant Human Hepatocyte Growth Factor in Patients with Acute Spinal Cord Injury: A Double-Blind, Randomized Clinical Trial of Safety and Efficacy
}

\author{
Narihito Nagoshi, ${ }^{1}$ Osahiko Tsuji, ${ }^{1}$ Kazuya Kitamura, ${ }^{2}$ Kota Suda, ${ }^{3}$ Takeshi Maeda, ${ }^{4}$ Yoshiyuki Yato, ${ }^{5}$ \\ Takayuki Abe, ${ }^{6,7}$ Daichika Hayata, ${ }^{8}$ Morio Matsumoto, ${ }^{1}$ Hideyuki Okano, ${ }^{9}$ and Masaya Nakamura ${ }^{1}$
}

\begin{abstract}
Spinal cord injury (SCI) is an abrupt traumatic injury that leads to permanent functional loss, and no practical treatment is available. We have developed pharmaceutical recombinant human hepatocyte growth factor (KP-100), and its efficacy for SCI has been verified using animal models. The purpose of this study was to evaluate the safety and efficacy of intrathecal KP-100 administration for SCI patients in the acute phase. This investigation was a multi-center, randomized, double-blind study. Subjects with modified Frankel grade A/B1/B2 at $72 \mathrm{~h}$ after SCI were included. KP-100 was administered intrathecally. Subjects were followed up for 168 days after the first administration. Outcomes were evaluated using American Spinal Injury Association (ASIA) scores and subjected to analysis of covariance. Our results demonstrated that the subjects did not show any serious adverse events caused by KP-100. Forty-three subjects underwent neurological function testing (26 in KP-100 group; 17 in placebo group), which revealed that KP-100 contributed to motor improvement at Days $140(p=0.050)$ and $168(p=0.079)$. In the subset of subjects with Frankel grade A, the proportions of subjects who gained at least 1 point on their lower-extremity motor scores were $33.3 \%(5 / 15)$ and $6.3 \%(1 / 16)$ in the KP-100 and placebo groups, respectively ( $p=0.083)$. Therefore, KP-100 has the potential to be useful and beneficial for SCI patients during the acute phase. However, this was a phase I/II trial and did not definitely address the question of efficacy; a larger phase III trial would be required to assess the efficacy.
\end{abstract}

Keywords: clinical trial; hepatocyte growth factor; SCI

\section{Introduction}

S PINAL CORD INJURY (SCI) is an abrupt, devastating event that leads to permanent loss of neurological function. SCI management requires considerable expense for years, resulting in an economic burden. Currently, the standard strategy for SCI treatment is to perform spinal fixation surgery, and recent SCI guidelines recommend early surgical decompression for functional improvement. ${ }^{1}$ However, the surgical intervention cannot produce sufficient recovery, especially in patients with complete motor loss.
SCI first starts from primary damage caused by an initial mechanical impact to the spinal cord, and subsequent persistent compression triggers further expansion of lesional extent and neural cell death, representing secondary damage. ${ }^{2}$ Several pre-clinical studies demonstrated favorable neurological restoration by administering neurotrophic factors to alleviate secondary damage, ${ }^{3-5}$ but decisive clinical treatment using these factors has not yet been established. Although methylprednisolone sodium succinate (MPSS) administration is the only approved procedure for acute stage SCI and is recommended as a treatment

\footnotetext{
${ }^{1}$ Department of Orthopedic Surgery, ${ }^{7}$ Biostatistics, Clinical and Translational Research Center, and ${ }^{9}$ Department of Physiology, Keio University School of Medicine, Tokyo, Japan.

${ }^{2}$ Department of Orthopedic Surgery, Saiseikai Yokohama-shi Tobu Hospital, Kanagawa, Japan.

${ }^{3}$ Department of Orthopedic Surgery, Hokkaido Spinal Cord Injury Center, Hokkaido, Japan.

${ }^{4}$ Department of Orthopedic Surgery, Spinal Injuries Center, Fukuoka, Japan.

${ }^{5}$ Department of Orthopedic Surgery, Murayama Medical Center, National Hospital Organization, Tokyo, Japan.

${ }^{6}$ Faculty of Data Science, Yokohama City University School of Data Science, Kanagawaken, Japan.

${ }^{8}$ Kringle Pharma, Inc., Osaka, Japan.

(C) Narihito Nagoshi et al., 2020; Published by Mary Ann Liebert, Inc. This Open Access article is distributed under the terms of the Creative Commons License (http://creativecommons.org/licenses/by/4.0), which permits unrestricted use, distribution, and reproduction in any medium, provided the original work is properly credited.
} 
option in a recent guideline, ${ }^{6,7}$ its efficacy and safety still remain controversial. 8,9

Hepatocyte growth factor (HGF) was first identified as a powerful mitogen for mature hepatocytes. ${ }^{10} \mathrm{HGF}$ binds to its specific receptor c-Met, and this signaling pathway plays significant roles in protection, regeneration, and homeostasis in various organs. ${ }^{11}$ In the diseased central nervous system (CNS), intrathecally administered recombinant human HGF (rhHGF) promoted neural cell survival and contributed to functional restoration in cerebral ischemia $^{12,13}$ and higher survival rates in amyotrophic lateral sclerosis (ALS). ${ }^{14}$ Regarding SCI, in our pre-clinical studies, HGF exerted a neuroprotective effect and enhanced angiogenesis, which resulted in axonal regeneration and motor functional recovery. ${ }^{15,16}$ Next, we established a more severe contusion model of cervical SCI in non-human primates using common marmosets (Callithrix jacchus), which presented almost no recovery of upper extremities during the follow-up period; however, despite this severity, the animals gained significant functional improvement after intrathecal rhHGF administration. ${ }^{17}$ These results encouraged us to use rhHGF for patients with SCI and achieve our clinical application project.

Our group has refined a natural variant of HGF (Kringle Pharma development code: KP-100) with a five-amino-acid deletion ${ }^{18}$ and sought to apply this agent to CNS diseases. A completed phase I trial for ALS has certified its tolerability. ${ }^{19}$ Therefore, the current study aimed to evaluate the safety and efficacy of intrathecal KP-100 administration in the acute phase of SCI.

\section{Methods}

\section{Trial design}

This investigation was a multi-center, randomized, double-blind, placebo-controlled, parallel-group study. The randomization was imbalanced at 2:1, predominantly in the KP-100 group. Patient registration and treatment took place at three high-volume SCI centers. The rehabilitation program was unified among the institutions. The study period was from June 2014 to May 2018. This study was registered at ClinicalTrials.gov (Identifier: NCT02193334). The study was implemented according to the Consolidated Standards of Reporting Trials (CONSORT) 2010 Statement. $^{20}$

\section{Patients}

Patients meeting the following criteria were initially selected for pre-registered inclusion: (1) cervical SCI within the past $60 \mathrm{~h}$ and a modified Frankel grade of A/B1/B2 at $48-60 \mathrm{~h}$ after injury; (2) informed consent provided; and (3) being 18-75 years old at the time of consent. In addition, all of the following criteria were met: (4) SCI within the past $78 \mathrm{~h}$ and modified Frankel grade of A/B1/B2 at $72 \mathrm{~h}$ after injury; and (5) written informed consent provided. Modified Frankel grade is shown in Table S1. ${ }^{21,22}$ The exclusion criteria were as follows: (1) The injury site was C1-C2 or C2-C3; (2) the patient could not start the rehabilitation necessary for recovery of function at an early stage; (3) it was not possible to administer the first dose of the study drug within $78 \mathrm{~h}$ after SCI; (4) the patient had a history of SCI or abnormal findings in the spinal cavity or marked breakdown of the meninges; (5) efficacy and safety could not be evaluated properly for reasons such as concurrent multiple external trauma or concurrent organ injury; (6) high-dose steroid therapy had been administered within 1 month before the main registration; (7) the patient had a disease such as serious liver disorder, kidney disease, heart disease, blood dyscrasia, metabolic disease, or infections requiring systemic therapy; (8) the patient had a history of malignant tumor; (9) the patient had participated in a clinical study or research of pharmaceuticals or medical devices within 1 month before registration; (10) the patient had allergies to drugs that would be used; (11) administration of the study drug to the site of SCI would be inappropriate for a reason such as intrathecal infection or intrathecal mass; (12) problems with the patient's ability to give informed consent in person; (13) the patient was breastfeeding or may be pregnant; and (14) it was inappropriate for the patient to be included in the study in the opinion of the investigator.

\section{Interventions}

The investigation drug was a lyophilized preparation containing KP-100. The drug $(0.6 \mathrm{~mL})$ was prepared at a concentration of $1 \mathrm{mg} / \mathrm{mL}$. A lyophilized formulation not containing KP-100 was prepared for the control drug. After patient registration, the first dose of the study drug (Day 0) was administered within $78 \mathrm{~h}$ after injury. Thereafter, the study drug was intrathecally administered through lumbar puncture procedures once a week for a total of 5 times. The observation period was from 2 days after the final dose (Day 30) until the final observation date (Day 168). All evaluated subjects were treated as inpatients during this period.

\section{Outcomes}

Adverse events were evaluated during the follow-up period. Efficacy was evaluated in change from baseline in American Spinal Injury Association (ASIA) motor and sensory scores. At baseline, we examined the ASIA impairment scale (AIS). We also investigated the time course of modified Frankel grade ${ }^{22}$ and KP-100 plasma and cerebrospinal fluid concentrations. Both the attending physicians on site and the physicians in charge of this trial discussed the severity of the SCI before registration and determined the modified Frankel grade.

\section{Sample size}

The assumptions for the standard deviation and intergroup difference in the mean changes in the ASIA motor score were based on previous references. ${ }^{23,24}$ In those studies, there was a difference of 15.3 points between the treatment and placebo groups in the entire population. However, not only patients with Frankel grades A/B $(62.5 \%)$, but also those with grades C/D were included (37.5\%) in the trial. Considering the 15.3 point intergroup difference is a weighted average of $X$ point difference in the Frankel grade $A / B$ and 0 point difference in Frankel grade $\mathrm{C} / \mathrm{D}$ in their trial, $\mathrm{X}=24$ points intergroup difference was expected in the Frankel grade A/B only (15.3/ $0.625=24.4$ ). Assuming that the changes from baseline ASIA motor scores at Day 168 had an intergroup difference of 24 points with a standard deviation of 25 points, the minimum sample size achieving a power $\geq 80 \%$ for tests based on approximation to a standard distribution (two-sided significance level of 5\%) with a placebo:active drug ratio of 1:2 was $\sim 42$ subjects. As the motor score analysis set in this study was the full analysis set (FAS), we selected a target sample size of 16 subjects in the placebo group and 32 subjects in the KP-100 group, assuming exclusion of $10 \%$ of registered subjects.

\section{Randomization and blinding}

The assignment table was randomly assigned to investigational drugs so that four boxes of the KP-100 group and two boxes of the placebo group were randomized among six boxes (one block). KP-100 and placebo formulations were indistinguishable in appearance and were unknown to all individuals involved in this study other than the assignment manager.

\section{Statistical methods}

ASIA scores are presented as point estimates \pm standard error, and other parameters are presented as the means \pm standard deviation. Baseline demographics and pre-operative scores in the two groups were compared by unpaired $t$ test for continuous variables and a $\chi^{2}$ test for categorical variables. 
For the primary end-point (changes in ASIA motor score at Day 168), missing values were imputed using the last observation carried forward (LOCF) method. The changes from baseline in ASIA motor score at Day 168 were analyzed using analysis of covariance (ANCOVA) with the reaction variable, treatment group, and baseline ASIA motor score as covariates. The same ANCOVA was performed for the ASIA sensory score and other time points for the ASIA motor score. A two-sided significance level of 5\% was used for analysis, and a two-sided $95 \%$ confidence coefficient was used for confidence intervals.

\section{Data availability}

De-identified participant data will be available to qualified investigators upon request to the corresponding author.

\section{Results}

Figure 1shows a diagram of patient flow in this study. Fortyeight patients were pre-registered; however, three were excluded because two exhibited worsening conditions and were transferred to other hospitals, and one recovered to modified Frankel grade B3. After randomization, 45 patients were divided into the KP-100 group $(n=28)$ or the placebo group $(n=17)$, and safety analysis was performed for these groups as the safety analysis set (SAS). Prior to analysis of efficacy, two additional patients were excluded from the $\mathrm{KP}-100$ group because they experienced respiratory dysfunction after the first drug administration and were unable to continue the intervention. Finally, analysis was performed for 43 patients with FAS.

\section{Safety results}

The frequency of adverse reactions in the KP-100 and placebo groups was $7.1 \%(2 / 28)$ and $5.9 \%(1 / 17)$, respectively. Adverse reactions comprised abnormal hepatic function (increment in the alkaline phosphatase, lactate dehydrogenase, aspartate aminotransferase [AST], and alanine aminotransferase [ALT] levels; $n=1)$, musculoskeletal discomfort $(n=1)$, increased hepatic enzymes (increment in the AST and ALT levels; $n=1$ ), and spinal cord edema $(n=1)$ in the KP-100 group and lower gastrointestinal hemorrhage $(n=1)$ and abnormal hepatic function $(n=1)$ in the placebo group.

The serious adverse events encompassed five occurrences in four subjects in the KP-100 group (one occurrence each of asphyxia, septic shock, and breast cancer and two occurrences of aspiration pneumonia) and one occurrence in one subject in the placebo group (cerebral infarction). In the KP-100 group, one subject died of aspiration pneumonia. A causal relationship with the administered drug was ruled out for every serious adverse event. Anti-KP-100 antibody was not detected in the blood or cerebrospinal fluid of any subjects.

\section{Demographics and baseline characteristics for efficacy analysis}

Table 1 presents the demographic data for FAS. For modified Frankel grade prior to drug injection, the frequency of Frankel grade A was the highest in both groups (57.7\% in KP-100, $94.1 \%$ in placebo). Cases with incomplete sensory loss were more predominant in the KP-100 group than in the placebo group (B1: $15.4 \%$ vs. $5.9 \%$, B2: $26.9 \%$ vs. $0.0 \%, p=0.022)$. Similar results were observed using AIS, wherein the frequency of patients with the AIS A classification was lower in the KP-100 group $(57.7 \%$ vs. $94.1 \%)$ whereas that of those with AIS B classification was higher (42.3\% vs. $5.9 \%$, $p=0.024)$. In the KP-100 group, baseline ASIA motor and sensory scores were lower $(10.6 \pm 11.0$ vs. $14.5 \pm 15.0)$ and higher $(48.5 \pm$ 28.7 vs. $39.1 \pm 19.8$ ), respectively. However, these differences were non-significant (motor: $p=0.331$, sensory: $p=0.246$ ).

\section{Efficacy results}

At several time points, the changes from baseline in the ASIA motor score were different between the KP-100 and placebo groups, with a two-sided significance level of $p<0.100$ at Days 84 $(10.1 \pm 2.0$ vs. $4.0 \pm 2.5, p=0.063), 112(10.6 \pm 2.2$ vs. $4.2 \pm 2.7$, $p=0.075), 140(12.6 \pm 2.4$ vs. $4.8 \pm 3.0, p=0.050)$, and 168 $(12.4 \pm 2.4$ vs. $5.3 \pm 3.0, p=0.079)$ (Fig. $2 \mathrm{~A}$ ). The changes from

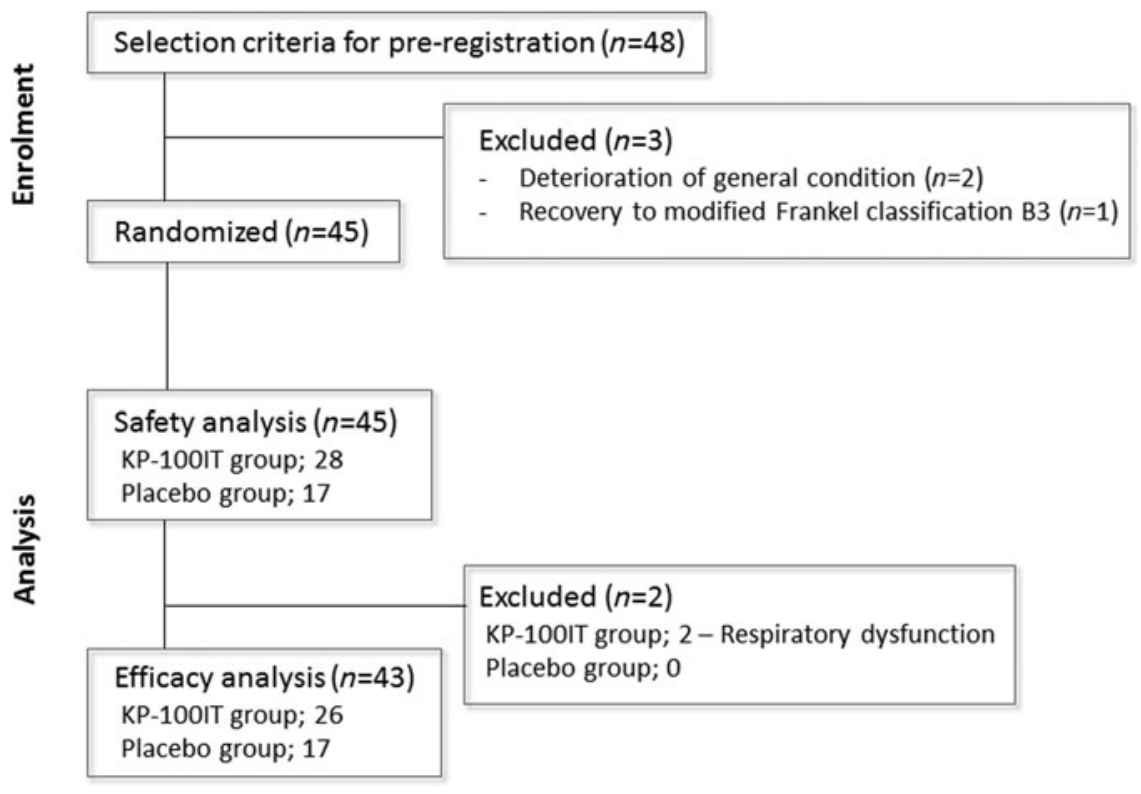

FIG. 1. A diagram of patient flow. 
Table 1. Demographic Characteristics of Patients in the KP-100 and Placebo Groups (Full Analysis Set)

\begin{tabular}{lccc}
\hline & $\begin{array}{c}\text { KP-100 } \\
(\mathrm{n}=26)\end{array}$ & $\begin{array}{c}\text { Placebo } \\
(\mathrm{n}=17)\end{array}$ & $\mathrm{p}$ value \\
\hline Age (y) & $59.3 \pm 12.5$ & $58.2 \pm 15.3$ & 0.800 \\
Sex (\% male) & 76.9 & 82.4 & 1.000 \\
Race (\% East Asians) & 100.0 & 100.0 & 1.000 \\
Modified Frankel grade & & & 0.022 \\
$\quad$ A & 57.7 & 94.1 & \\
$\quad$ B1 & 15.4 & 5.9 & \\
$\quad$ B2 & 26.9 & 0.0 & \\
ASIA impairment scale & & & 0.024 \\
$\quad$ A & 57.7 & 94.1 & \\
$\quad$ B & 42.3 & 5.9 & \\
Baseline ASIA motor score & $10.6 \pm 11.0$ & $14.5 \pm 15.0$ & 0.331 \\
Baseline ASIA sensory score & $48.5 \pm 28.7$ & $39.1 \pm 19.8$ & 0.246 \\
Surgical cases (\%) & 61.5 & 82.4 & 0.187 \\
\hline
\end{tabular}

ASIA, American Spinal Injury Association.

baseline in the ASIA sensory score were almost comparable at Days $84(22.1 \pm 7.3$ vs. $26.5 \pm 9.1, p=0.709)$ and 168 (34.5 \pm 9.8 vs. $34.0 \pm 12.2, p=0.977$ ) (Fig. 2B). Missing values were imputed at three and one time points in the KP-100 and placebo groups, respectively.

When changes in ASIA motor scores were investigated separately for upper and lower extremities, slightly better improvement was observed in upper extremities in the KP-100 group from Day 42, and final scores were $4.9 \pm 0.9$ and $4.3 \pm 1.2$ in the KP-100 and placebo groups, respectively (Fig. 2C), but were not significantly different at any time point. Alternatively, remarkable score recovery occurred in the lower extremity during the observational period, with significant differences at Days 140 (7.5 \pm 1.8 vs. $1.1 \pm 2.2, p=0.031)$ and 168 (7.3 \pm 1.9 vs. $1.3 \pm 2.3, p=0.049)$ (Fig. $2 \mathrm{D})$.
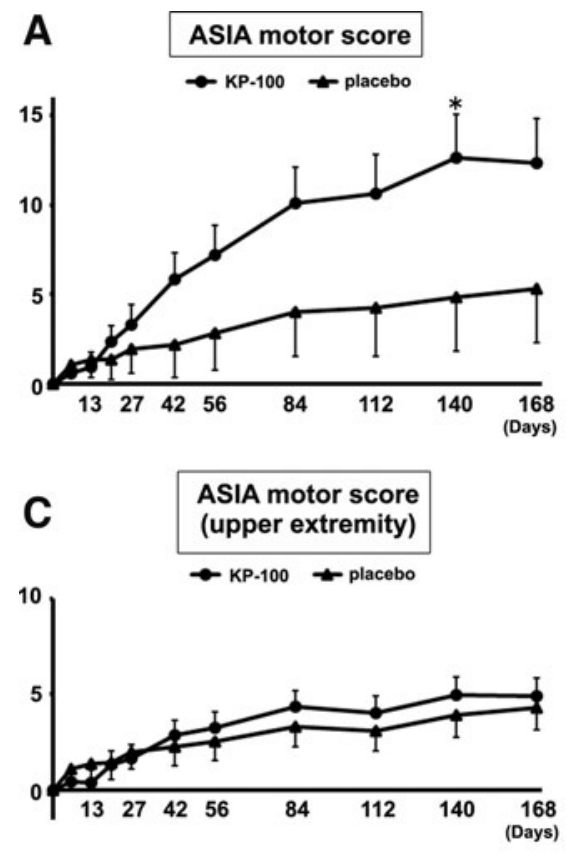

Because the frequency of the population with modified Frankel grade B was higher in the KP-100 group at baseline (Table 1), subanalysis was performed on subjects with Frankel grade A only. The KP-100 and placebo groups contained 15 and 16 subjects, respectively. There was no significant difference between the groups in age $(p=0.744)$ or gender $(p=1)$. From Day 20 until the final follow-up, ASIA motor scores were $8.8 \pm 3.0$ and $5.9 \pm 2.8$ in the KP-100 and placebo groups, respectively, at Day 168 $(p=0.504)$ (Fig. 3A). In ASIA sensory score analysis, both groups showed similar restoration without significant differences ( $p=0.977$ on Day 168) (Fig. 3B). When dividing the total motor scores into upper and lower extremities, changes in upperextremity scores were almost comparable between the groups (Fig. 3C). Regarding the lower extremities, the final scores were $5.4 \pm 2.1$ and $1.4 \pm 2.0$ in the KP-100 and placebo groups, respectively ( $p=0.171$ ) (Fig. 3D). The proportion of subjects who gained at least one point on motor scores in the lower extremities was $33.3 \%(5 / 15)$ and $6.3 \%(1 / 16)$ in the KP-100 and placebo groups, respectively $(p=0.083)$.

Figure 4shows the time course of modified Frankel grade in subjects with modified Frankel grade A at baseline. The percentage of subjects who improved by at least one modified Frankel grade was $46.7 \%$ (7/15 subjects) and $31.3 \%$ (5/16 subjects) in the KP-100 and placebo groups, respectively, at Day $168(p=0.379)$. The percentages of subjects who improved to grade $\mathrm{C} 1$ were $26.7 \%$ (4/15 subjects) and 6.3\% (1/16 subjects) in the KP-100 and placebo groups, respectively, at the final observation $(p=0.172)$.

\section{Discussion}

In the present study, favorable motor functional recovery was observed in patients who underwent intrathecal KP-100 administration during acute stage SCI. In particular, higher motor scores were observed in the KP-100 group than in the placebo group in the
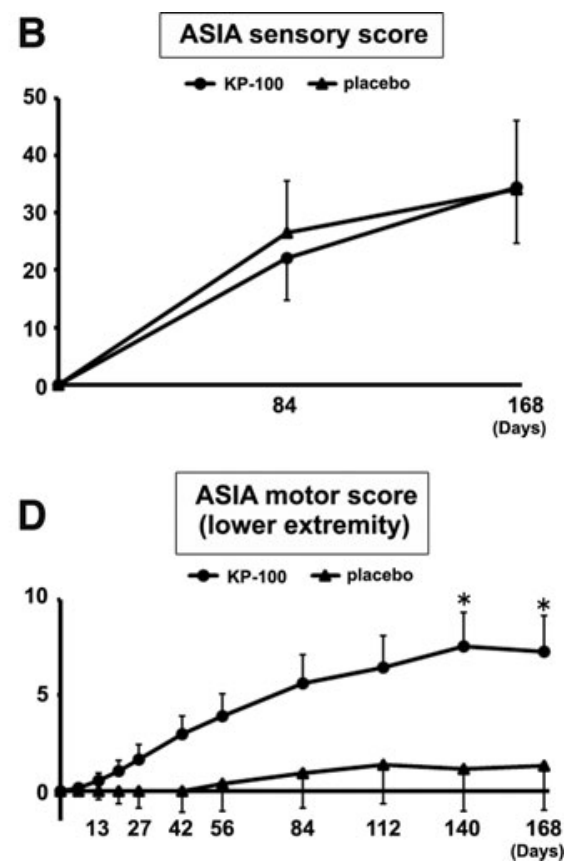

FIG. 2. Change in American Spinal Injury Association (ASIA) scores for subjects in the KP-100 ( $n=26)$ and placebo groups $(n=17)$, using the full analysis set. (A) Motor score; (B) sensory score; (C) motor score in upper extremities; (D) motor score in lower extremities. ${ }^{*} p<0.05$. 

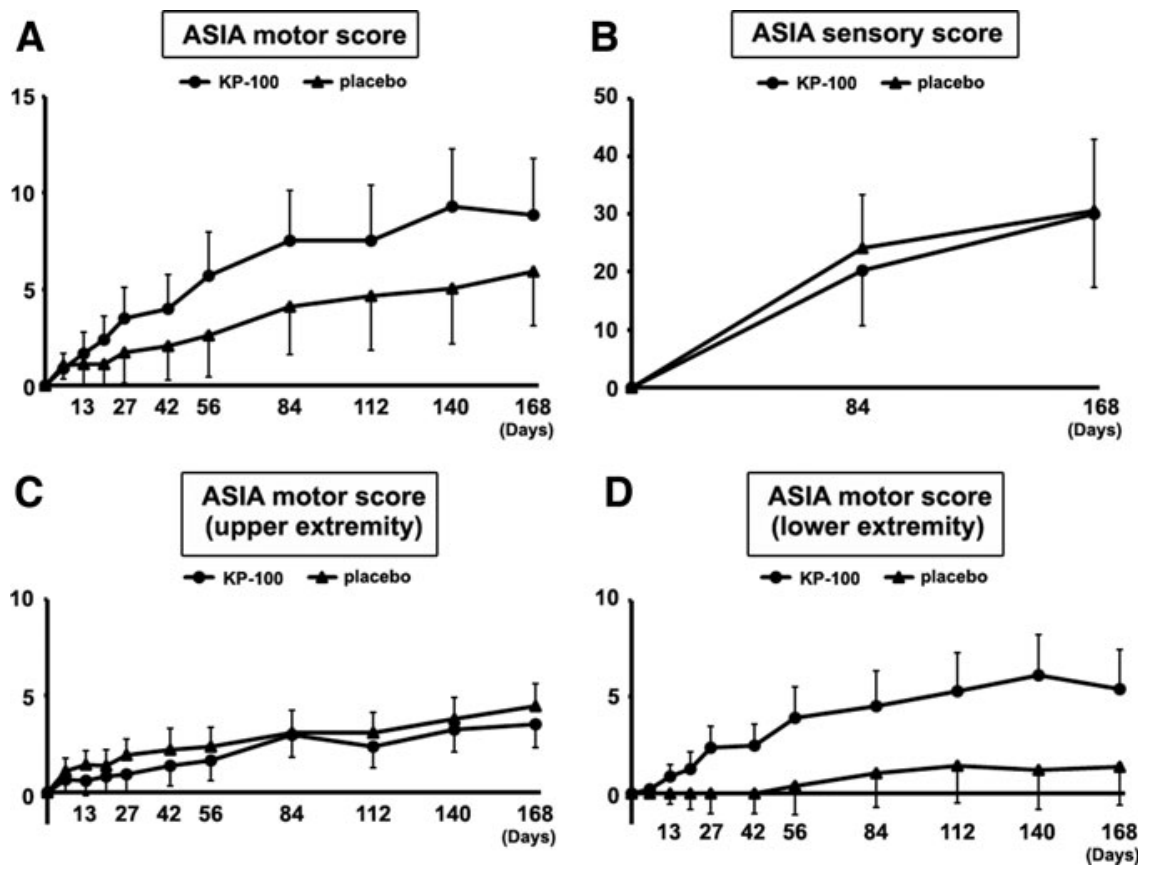

FIG. 3. Change in American Spinal Injury Association (ASIA) scores for subjects in the KP-100 $(n=15)$ and placebo groups $(n=16)$ with modified Frankel grade A. (A) Motor score; (B) sensory score; (C) motor score in upper extremities; (D) motor score in lower extremities.

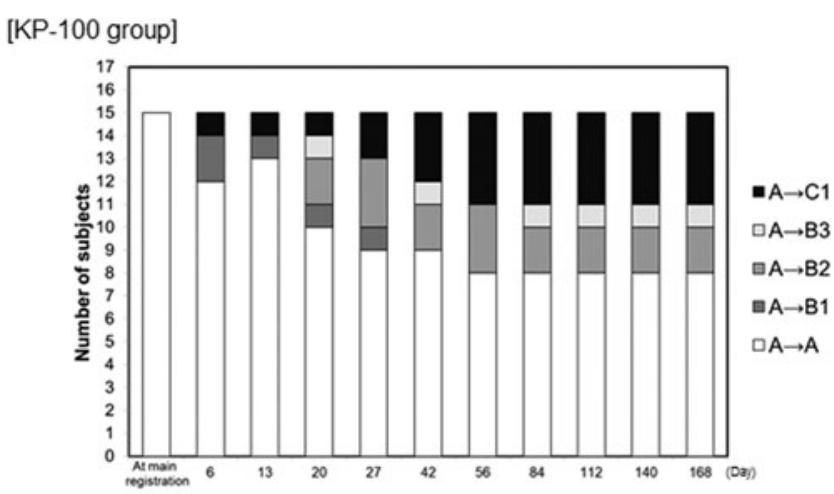

[Placebo group]

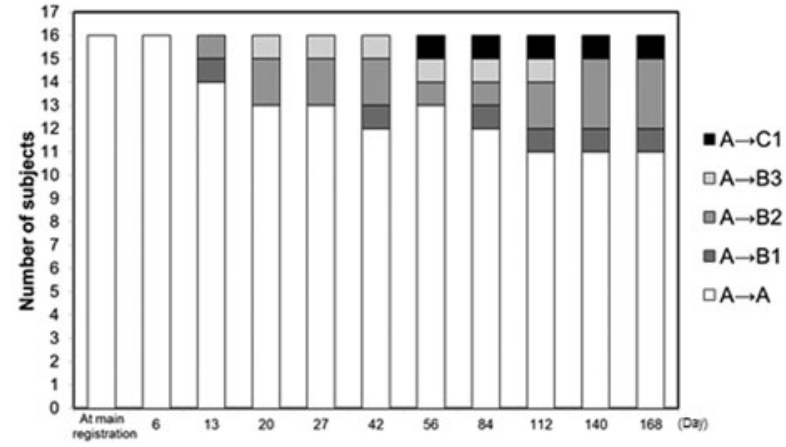

FIG. 4. Changes in modified Frankel grades during the observational period. lower extremities. Regarding safety, KP-100 administration did not cause any harmful adverse events during the observational period. Together, considering both safety and efficacy, KP-100 could be a beneficial drug for SCI, which is typically considered an irreparable traumatic injury.

The lateral corticospinal tract in the spinal cord consists of the voluntary motor pathway, and the area that controls upperextremity function is in the medial part of the tract, whereas the area for lower-extremity function is more lateral. During contusion injury, severe damage predominantly occurs in the central parts of the spinal cord from the strong external force. ${ }^{2}$ Therefore, damage to the central part of the cord inevitably leads to more severe paralysis in the upper extremities than in the lower extremities. This pathological condition could explain the different degrees of motor recovery between the upper and lower extremities in the current study. In our pre-clinical studies, HGF facilitated neuroprotection, preventing the expansion of secondary damage after SCI. ${ }^{15,16}$ This efficacy had the potential to increase motor recovery in the lower extremities among SCI patients, because its anatomical place in the pyramidal tracts is distant from the lesion epicenter. Our study registered only patients with severe paralysis who were expected to have a large lesional area. However, if we investigated patients with less severe SCI, such as those with modified Frankel grade C or D, HGF administration might provide motor functional recovery even for upper extremities, by further reducing the smaller lesional area.

MPSS is reportedly efficacious when administered within $8 \mathrm{~h}$ after injury. ${ }^{6}$ We reviewed that study in detail and found that the neurological function assessment prior to drug administration occurred upon admission. However, the neurological assessment within the first $24 \mathrm{~h}$ after SCI is unreliable because of spinal shock, conditional instability, or concomitant brain injury. ${ }^{25}$ These events could confound the true severity status because some populations achieve spontaneous improvement even if the initial examination 
identified complete motor loss. In fact, according to Brown and coworkers, ${ }^{26}$ patients with complete motor loss within $24 \mathrm{~h}$ after injury still exhibited subsequent changes in muscle strength. On the other hand, Crozier and coworkers ${ }^{27}$ demonstrated that among the SCI patients with Frankel grade B, motor neurological recovery was markedly limited if preservation of pain sensation was not detected at $72 \mathrm{~h}$ post- injury. Therefore, assessment at $72 \mathrm{~h}$ provided a more precise prediction of later severity. Given this pathology, we evaluated the neurological status $72 \mathrm{~h}$ after injury and then enrolled patients whose modified Frankel grade was A, B1 or B2 (without pain sensation). Because patients with these classifications rarely presented motor recovery, ${ }^{27,28}$ we could prevent the detection of false drug effects, which were concealed by subjects showing spontaneous restoration. Therefore, we adopted the modified Frankel classification instead of the ASIA impairment classification that cannot classify sensory incompleteness in detail.

Compared with previous clinical trials, our study design configured the longest duration between the injury and agent administration, which is likely to have provided an extremely unfavorable environment for the spinal cord regeneration. ${ }^{6,29-31}$ Even in that severe condition, delayed KP-100 administration exerted greater motor improvement in the lower extremities in the current study. In contrast to this notable finding, neurological recovery in the upper extremities was limited in the KP-100 group. However, our preclinical results using a marmoset cervical SCI model demonstrated significant motor restoration in their upper limbs when administering the drug right after the injury. ${ }^{17}$ Therefore, HGF administered as early as possible can be expected to prevent the further enlargement of secondary damage, and to achieve greater improvement in motor function by preserving a wide range of spinal cord tissue.

Our group will soon begin transplantation therapy using induced pluripotent stem cells (iPSCs) for SCI patients in the subacute phase. $^{32}$ The aim of this clinical research is to evaluate the outcomes of cell transplantation as a single therapeutic intervention. In the future, initially administering KP-100 is ideal for the purpose of reducing secondary damage, followed by transplanting iPSC-derived neural precursor cells into the injured spinal cord where the environment is more advantageous for cell survival and regeneration.

This study has several limitations that should be described. First, our study design lacked dynamic allocation for randomization, which resulted in an imbalance of neurological severity at initial examination between the groups. Consequently, significantly more patients with incomplete paralysis (Frankel grade B) were registered in the KP-100 group, which had the capability of achieving superior neurological improvement compared with the placebo group. Accordingly, subanalysis was necessary to compare the results, by targeting subjects with modified Frankel grade A, but statistical power was reduced because of the small sample size. In the future, a larger scale multi-center study should be performed to validate KP-100's efficacy. Second, our inclusion criteria were limited to subjects with complete motor loss. The next step is to evaluate the drug effect for patients with less severe injuries, to broaden the utility of this agent. Finally, there were no standardized indications for surgical methods, and they were decided at the discretion of each institute. These factors might influence the results in the analyzed subjects.

In conclusion, given its ease of administration, potential efficacy, and lack of significant safety problems, KP-100 could be a convenient, useful therapeutic agent for SCI patients in the acute phase.

\section{Acknowledgments}

We thank the members of the Independent Data Monitoring Committee (Fumihiro Tajima MD, PhD, Wakayama Medical University; Yasuo Ito MD, PhD, Kobe Red Cross Hospital; and Shinsuke Katoh MD, PhD, The University of Tokushima). We thank to the study's medical adviser Hiroshi Funakoshi MD, PhD (Asahikawa Medical University). We also thank Miki Komatsu, MD, PhD (Hokkaido Spinal Cord Injury Center); Hiroaki Sakai, $\mathrm{MD}, \mathrm{PhD}$, (Spinal Injuries Center) and Kanehiro Fujiyoshi, MD, $\mathrm{PhD}$, (Murayama Medical Center, National Hospital Organization) for clinical contributions to this trial.

\section{Informed Consent}

This study was approved by our institution's review boards, and all subjects gave written informed consent for inclusion.

Classification of evidence: Class I

Trial registration number

Phase I/II Study of KP-100IT in Acute Spinal Cord Injury

NCT02193334

https://clinicaltrials.gov/ct2/show/NCT02193334

\section{Funding Information}

This research was supported by the Japan Agency for Medical Research and Development under Grant Numbers JP16im0110701 and JP18nk0101210.

\section{Author Disclosure Statement}

No competing financial interests exist.

\section{Supplementary Material}

Supplementary Table S1

\section{References}

1. Fehlings, M.G., Tetreault, L.A., Wilson, J.R., Aarabi, B., Anderson, P., Arnold, P.M., Brodke, D.S., Burns, A.S., Chiba, K., Dettori, J.R., Furlan, J.C., Hawryluk, G., Holly, L.T., Howley, S., Jeji, T., KalsiRyan, S., Kotter, M., Kurpad, S., Marino, R.J., Martin, A.R., Massicotte, E., Merli, G., Middleton, J.W., Nakashima, H., Nagoshi, N., Palmieri, K., Singh, A., Skelly, A.C., Tsai, E.C., Vaccaro, A., Yee, A., and Harrop, J.S. (2017). A clinical practice guideline for the management of patients with acute spinal cord injury and central cord syndrome: recommendations on the timing $(</=24$ hours versus $>24$ hours) of decompressive surgery. Global Spine J. 7, 195S-202S.

2. Tator, C.H., and Fehlings, M.G. (1991). Review of the secondary injury theory of acute spinal cord trauma with emphasis on vascular mechanisms. J. Neurosurg. 75, 15-26.

3. Grill, R., Murai, K., Blesch, A., Gage, F.H., and Tuszynski, M.H. (1997). Cellular delivery of neurotrophin-3 promotes corticospinal axonal growth and partial functional recovery after spinal cord injury. J. Neurosci. 17, 5560-5572.

4. Jakeman, L.B., Wei, P., Guan, Z., and Stokes, B.T. (1998). Brainderived neurotrophic factor stimulates hindlimb stepping and sprouting of cholinergic fibers after spinal cord injury. Exp. Neurol. 154, $170-184$.

5. Tuszynski, M.H., Gabriel, K., Gage, F.H., Suhr, S., Meyer, S., and Rosetti, A. (1996). Nerve growth factor delivery by gene transfer induces differential outgrowth of sensory, motor, and noradrenergic neurites after adult spinal cord injury. Exp. Neurol. 137, 157-173.

6. Bracken, M.B., Shepard, M.J., Collins, W.F., Holford, T.R., Young, W., Baskin, D.S., Eisenberg, H.M., Flamm, E., Leo-Summers, L., Maroon, J. and et al. (1990). A randomized, controlled trial of methylprednisolone or naloxone in the treatment of acute spinal-cord injury. Results of the Second National Acute Spinal Cord Injury Study. N. Engl. J. Med. 322, 1405-1411. 
7. Fehlings, M.G., Wilson, J.R., Tetreault, L.A., Aarabi, B., Anderson P., Arnold, P.M., Brodke, D.S., Burns, A.S., Chiba, K., Dettori, J.R., Furlan, J.C., Hawryluk, G., Holly, L.T., Howley, S., Jeji, T., KalsiRyan, S., Kotter, M., Kurpad, S., Kwon, B.K., Marino, R.J., Martin, A.R., Massicotte, E., Merli, G., Middleton, J.W., Nakashima, H., Nagoshi, N., Palmieri, K., Skelly, A.C., Singh, A., Tsai, E.C., Vaccaro, A., Yee, A., and Harrop, J.S. (2017). A clinical practice guideline for the management of patients with acute spinal cord injury: recommendations on the use of methylprednisolone sodium succinate. Global Spine J. 7, 203S-211S.

8. Fehlings, M.G., Wilson, J.R., and Cho, N. (2014). Methylprednisolone for the treatment of acute spinal cord injury: counterpoint. Neurosurgery 61, Suppl. 1, 36-42.

9. Hurlbert, R.J. (2014). Methylprednisolone for the treatment of acute spinal cord injury: point. Neurosurgery 61, Suppl. 1, 32-35.

10. Nakamura, T., Nishizawa, T., Hagiya, M., Seki, T., Shimonishi, M., Sugimura, A., Tashiro, K., and Shimizu, S. (1989). Molecular cloning and expression of human hepatocyte growth factor. Nature 342, 440-443.

11. Matsumoto, K., Funakoshi, H., Takahashi, H., and Sakai, K. (2014). HGF-met pathway in regeneration and drug discovery. Biomedicines 2, 275-300

12. Date, I., Takagi, N., Takagi, K., Kago, T., Matsumoto, K., Nakamura, T., and Takeo, S. (2004). Hepatocyte growth factor improved learning and memory dysfunction of microsphere-embolized rats. J. Neurosci. Res. 78, 442-453.

13. Tsuzuki, N., Miyazawa, T., Matsumoto, K., Nakamura, T., and Shima, K. (2001). Hepatocyte growth factor reduces the infarct volume after transient focal cerebral ischemia in rats. Neurol. Res. 23, 417-424.

14. Ishigaki, A., Aoki, M., Nagai, M., Warita, H., Kato, S., Kato, M., Nakamura, T., Funakoshi, H., and Itoyama, Y. (2007). Intrathecal delivery of hepatocyte growth factor from amyotrophic lateral sclerosis onset suppresses disease progression in rat amyotrophic lateral sclerosis model. J. Neuropathol. Exp. Neurol. 66, 1037-1044.

15. Kitamura, K., Fujiyoshi, K., Yamane, J., Toyota, F., Hikishima, K., Nomura, T., Funakoshi, H., Nakamura, T., Aoki, M., Toyama, Y., Okano, H., and Nakamura, M. (2011). Human hepatocyte growth factor promotes functional recovery in primates after spinal cord injury. PloS One 6, e27706.

16. Kitamura, K., Iwanami, A., Nakamura, M., Yamane, J., Watanabe, K., Suzuki, Y., Miyazawa, D., Shibata, S., Funakoshi, H., Miyatake, S., Coffin, R.S., Nakamura, T., Toyama, Y., and Okano, H. (2007). Hepatocyte growth factor promotes endogenous repair and functional recovery after spinal cord injury. J. Neurosci. Res. 85, 2332-2342.

17. Kitamura, K., Iwanami, A., Iwai, H., Toyama, Y., Matsumoto, M., Okano, H., and Nakamura, M. (2016). Therapeutic time window and preclinical efficacy of intrathecal administration of recombinant human hepatocyte growth factor for acute spinal cord injury. J. Spine Res. 7, 934-939.

18. Shima, N., Tsuda, E., Goto, M., Yano, K., Hayasaka, H., Ueda, M., and Higashio, K. (1994). Hepatocyte growth factor and its variant with a deletion of five amino acids are distinguishable in their biological activity and tertiary structure. Biochem. Biophys. Res. Commun. 200, 808-815.

19. Warita, H., Kato, M., Asada, R., Yamashita, A., Hayata, D., Adachi, K., and Aoki, M. (2019). Safety, tolerability, and pharmacodynamics of intrathecal injection of recombinant human HGF (KP-100) in subjects with amyotrophic lateral sclerosis: a phase I trial. J. Clin. Pharmacol. 59, 677-687.

20. Moher, D., Hopewell, S., Schulz, K.F., Montori, V., Gotzsche, P.C., Devereaux, P.J., Elbourne, D., Egger, M., and Altman, D.G. (2010). CONSORT 2010 explanation and elaboration: updated guidelines for reporting parallel group randomised trials. BMJ 340, c869.

21. Frankel, H.L., Hancock, D.O., Hyslop, G., Melzak, J., Michaelis, L.S., Ungar, G.H., Vernon, J.D., and Walsh, J.J. (1969). The value of postural reduction in the initial management of closed injuries of the spine with paraplegia and tetraplegia. I. Paraplegia 7, 179-192.

22. Hayashi, T., Kawano, O., Sakai, H., Ideta, R., Ueta, T., Maeda, T., Mori, E., Yugue, I., Takao, T., Masuda, M., Morishita, Y., and Shiba, K. (2013). The potential for functional recovery of upper extremity function following cervical spinal cord injury without major bone injury. Spinal Cord 51, 819-822.

23. Geisler, F.H., Coleman, W.P., Grieco, G., Poonian, D., and Sygen Study Group (2001). The Sygen multicenter acute spinal cord injury study. Spine 26, S87-S98.

24. Geisler, F.H., Dorsey, F.C., and Coleman, W.P. (1991). Recovery of motor function after spinal-cord injury-a randomized, placebocontrolled trial with GM-1 ganglioside. N. Engl J. Med. 324, 1829-1838.

25. Fawcett, J.W., Curt, A., Steeves, J.D., Coleman, W.P., Tuszynski, M.H., Lammertse, D., Bartlett, P.F., Blight, A.R., Dietz, V., Ditunno, J., Dobkin, B.H., Havton, L.A., Ellaway, P.H., Fehlings, M.G., Privat, A., Grossman, R., Guest, J.D., Kleitman, N., Nakamura, M., Gaviria, M., and Short, D. (2007). Guidelines for the conduct of clinical trials for spinal cord injury as developed by the ICCP panel: spontaneous recovery after spinal cord injury and statistical power needed for therapeutic clinical trials. Spinal Cord 45, 190-205.

26. Brown, P.J., Marino, R.J., Herbison, G.J., and Ditunno, J.F., Jr. (1991). The 72-hour examination as a predictor of recovery in motor complete quadriplegia. Arch. Phys. Med. Rehabil. 72, 546-548.

27. Crozier, K.S., Graziani, V., Ditunno, J.F., Jr., and Herbison, G.J. (1991). Spinal cord injury: prognosis for ambulation based on sensory examination in patients who are initially motor complete. Arch. Phys. Med. Rehabil. 72, 119-121.

28. Fukuda, F., and Ueta, T. (2001). Prediction of prognosis using modified Frankel classification in cervical spinal cord injured patients. Japanese Journal of Rehabilitation Medicine 38, 29-33.

29. Casha, S., Zygun, D., McGowan, M.D., Bains, I., Yong, V.W., and Hurlbert, R.J. (2012). Results of a phase II placebo-controlled randomized trial of minocycline in acute spinal cord injury. Brain 135, 1224-1236.

30. Fehlings, M.G., Theodore, N., Harrop, J., Maurais, G., Kuntz, C., Shaffrey, C.I., Kwon, B.K., Chapman, J., Yee, A., Tighe, A., and McKerracher, L. (2011). A phase I/IIa clinical trial of a recombinant Rho protein antagonist in acute spinal cord injury. J. Neurotrauma 28, 787-796.

31. Fehlings, M.G., Wilson, J.R., Frankowski, R.F., Toups, E.G., Aarabi, B., Harrop, J.S., Shaffrey, C.I., Harkema, S.J., Guest, J.D., Tator, C.H., Burau, K.D., Johnson, M.W., and Grossman, R.G. (2012). Riluzole for the treatment of acute traumatic spinal cord injury: rationale for and design of the NACTN Phase I clinical trial. J. Neurosurg. Spine 17, 151-156.

32. Tsuji, O., Sugai, K., Yamaguchi, R., Tashiro, S., Nagoshi, N., Kohyama, J., Iida, T., Ohkubo, T., Itakura, G., Isoda, M., Shinozaki, M., Fujiyoshi, K., Kanemura, Y., Yamanaka, S., Nakamura, M., and Okano, H. (2019). Concise review: laying the groundwork for a firstin-human study of an induced pluripotent stem cell-based intervention for spinal cord injury. Stem Cells 37, 6-13.

Address correspondence to:

Masaya Nakamura, MD, PhD

Department of Orthopedic Surgery

Keio University School of Medicine 35 Shinanomachi, Shinjukuku

Tokyo 160-8582

Japan

E-mail: masa@keio.jp 